\title{
1 Neurofilament light and tau in serum after head-impact exposure in soccer
}

2 Stian Bahr Sandmo ${ }^{\mathrm{a}, \mathrm{b}^{*}}$, Peter Filipcik ${ }^{\mathrm{c}}$, Martin Cente ${ }^{\mathrm{c}}$, Jozef Hanes ${ }^{\mathrm{c}}$,

3 Thor Einar Andersen ${ }^{\mathrm{a}}$, Truls Martin Straume-Naesheim ${ }^{\mathrm{a}, \mathrm{d}, \mathrm{e}}$, and Roald Bahr ${ }^{\mathrm{a}}$

$4 \quad{ }^{a}$ Oslo Sports Trauma Research Center, Department of Sports Medicine, Norwegian School of

5 Sport Sciences, Oslo, Norway

$6 \quad{ }^{b}$ Faculty of Medicine, University of Oslo, Oslo, Norway

7 'Institute of Neuroimmunology, Slovak Academy of Sciences, Bratislava, Slovakia

$8 \quad{ }^{d}$ Department of Orthopedic Surgery, Akershus University Hospital, Lørenskog, Norway

$9 \quad{ }^{e}$ Department of Orthopedic Surgery, Haugesund Rheumatism Hospital, Haugesund, Norway

$10 *$ Corresponding author:

11 Stian Bahr Sandmo, MD

12 Oslo Sports Trauma Research Center

13 Department of Sports Medicine, Norwegian School of Sport Sciences

14 PB 4014 Ullevål Stadion, NO-0806 Oslo, Norway

15 Telephone: +47 47666497

16 E-mail: s.b.sandmo@nih.no 


\section{Neurofilament light and tau in serum after head-impact exposure in soccer}

Introduction: Blood-based biomarkers can provide valuable information on the effects of repetitive head impacts in sports. This study investigated if repetitive headers or accidental head impacts in soccer could cause structural brain injury, detected as an increase in serum neurofilament light (NfL) or tau.

Methods: NfL and tau were measured in professional soccer players in pre-season. Then, the effect of three short-term exposures on biomarker levels was assessed:

(1) high-intensity exercise, (2) repetitive headers, and (3) head impacts in a match. Results: We analyzed 354 samples and observed no effects on NfL from any of the three short-term exposures. Tau levels rose significantly from baseline to $1 \mathrm{~h}$ after (1) high-intensity exercise $(\Delta 0.50 \mathrm{pg} / \mathrm{mL}, 95 \%$ CI $0.19-0.81, \mathrm{p}<0.01)$; the same was observed after (2) repetitive headers $(\Delta 0.29 \mathrm{pg} / \mathrm{mL}, 95 \%$ CI $0.10-0.48, \mathrm{p}<0.01)$, but not after (3) accidental head-impact incidents $(\Delta 0.36 \mathrm{pg} / \mathrm{mL}, 95 \% \mathrm{CI}-0.02-0.74, \mathrm{p}=0.06)$. The highest absolute values were seen $1 \mathrm{~h}$ after high-intensity exercise (mean $\pm \mathrm{SD}$, $1.92 \pm 0.83 \mathrm{pg} / \mathrm{mL})$.

Conclusion: NfL and tau in serum were unaffected by head impacts in soccer. Importantly, tau levels seem to rise in response to exercise, emphasizing the need for control groups. Our findings highlight important characteristics and limitations when using these biomarkers in sports.

Keywords: subconcussive, TBI, chronic traumatic encephalopathy, football, repetitive head impacts

\section{Introduction}

Head-impact exposure in contact sports remains a controversial issue because of the potential association with long-term neurological sequelae (1-3). Soccer is unique in that voluntary and unprotected use of the head, in the form of heading the ball, is an integral part of the game. This exposes players not only to repetitive head impacts in the relatively lower and typically asymptomatic range (4-6), but also to situations which may cause accidental and injurious head impacts of greater magnitudes (7). However, research on whether head-impact exposure in soccer is harmful for the brain has been inconclusive, as several key questions remain unanswered (8-10).

In order to further the understanding of different types of head-impact exposures and their potential consequences, it is central to develop objective diagnostic and prognostic 
tools (11). When the brain is exposed to accelerative forces, shearing of the white matter tracts accompanied by axonal injury might ensue $(12,13)$. This, again, can result in the release of structural neuronal proteins into the bloodstream. Consequently, several candidate blood-based biomarkers have been explored for use in traumatic brain injury (TBI) $(14,15)$, and some have been approved for clinical purposes $(16,17)$. However, previous studies have demonstrated the need for caution when using biomarkers in contact sports (18), as the confounding effects of e.g. exercise might limit their utility (19).

Two of the currently most promising blood biomarkers for use in contact sports are neurofilament light (NfL) and tau proteins, as recently demonstrated by Shahim et al. (20) using an ultrasensitive assay. Therefore, the main aim of this study was to explore if repetitive headers or accidental head impacts in soccer could cause short-term structural damage to the brain, detected as an increase in serum NfL or tau. In addition, as these two biomarkers have been shown also to reflect neurodegenerative processes $(21,22)$, we wanted to assess potential long-term effects on the brain by exploring the influence of previous head-impact exposure on pre-season resting NfL and tau serum concentration.

\section{Methods}

\section{Study design}

This study is based on a prospective cohort study designed to detect if minor head impacts in soccer could cause injury to the nervous tissue (19) or lead to measurable impairment in brain function $(23,24)$. During two consecutive seasons of the male Norwegian premier league, a total of 289 players in 2004 and 332 players in 2005 participated in the study, covering a total of 621 player seasons. To assess potential injury to the nervous tissue, blood samples were collected throughout the study period; data on changes in serum S100B have been published previously, where the effects of different conditions involving head impacts and/or exercise were assessed (19).

A subset of the blood samples were re-analyzed for the current study (table 1). This was done in order to assess acute changes in serum NfL and tau concentrations as a result of three different short-term exposures: 1) high-intensity exercise with no head-impact exposure, 2) low-intensity exercise with high head-impact exposure in the form of repetitive headers, and 3) head-impact incidents during a regular league match. All three conditions included blood samples before (baseline), $1 \mathrm{~h}$ after (B1), and $12 \mathrm{~h}$ after (B12) exposure. Furthermore, 
to assess changes in serum NfL and tau in relation to long-term effects, baseline samples from a subset of players from groups considered to be at low and high risk for cumulative head-impact exposure were re-analyzed.

\section{[Table 1 near here]}

The original study was approved by the Regional Committee for Medical and Health Research Ethics South (2004/14054) and the Data Inspectorate, and written informed consent was obtained from all participants. The approval and informed consent included the possibility of storing blood samples for later analyses, and the data have since been anonymized. For the purposes of this study, the Regional Committee for Medical and Health Research Ethics South East approved the analyses of the stored blood samples (2017/1104).

\section{Short-term exposure: (1) High-intensity exercise and (2) repetitive headers}

Players from three teams were invited to participate in two separate exercise sessions during the 2006 season. Blood samples were drawn once before the first session (baseline), and then within $1 \mathrm{~h}$ (B1) and the morning after (B12) both sessions. No other exercise was allowed between collection of the B1 and B12 samples. The sessions lasted 90 min each, were led by the team's regular coaching staff, and were completed on separate days. Both sessions were designed to closely mimic regular match situations, but with different characteristics related to head-impact exposure. Specifically, the high-intensity exercise session included regular soccer play, but with no heading of the ball. The repetitive headers session included multiple match-related drills where repetitive headers are commonly observed (e.g. corner kicks), but otherwise with low exercise intensity throughout the session. Importantly, the heading drills were specifically designed to reflect typical head impact magnitudes. On average, each player headed the ball 18.9 times (range 7.0-33.0) during the session. This average was approximately twice as high as the average number of headers per player in a regular league match (8.5, range $0-26)$, based on a manual count of 18 players during the 2004 season (19). 
2 Blood samples were drawn from the participants before the start of the 2004 and 2005 season

3 (baseline). Head impacts during regular league matches were then recorded by the team doctors or dedicated research personnel attending every match. Specifically, a head-impact incident was defined as and included if it fulfilled all three of the following criteria: (1) the player appeared to be hit in the head, neck or face, (2) the match was interrupted by the referee, and (3) the player remained lying down on the pitch for more than $15 \mathrm{~s}(7,25)$. After a head-impact incident, our research personnel were instructed to draw a blood sample within $1 \mathrm{~h}$ (B1) and within $12 \mathrm{~h}$ after (i.e. the next morning, B12) the match.

All head-impact incidents were classified as concussions or non-concussions based on the symptoms reported by the players themselves. Concussion was characterized as any short-lived impairment of neurological function after head trauma, as defined by the Concussion in Sports Group in Vienna 2001 (26). The concussion data were further crossreferenced with the reports from the team doctors to the league's injury surveillance system, which at the time included all time-loss injuries (27).

\section{Long-term effects: Previous head-impact exposure}

Players from the 2005 season $(n=280)$ were trichotomized into a low-risk, medium-risk and high-risk group. This was done using a questionnaire completed by the players (24), where they self-reported their previous head-impact exposure, including 1) the typical number of headers per match (never, 1-5 times, 6-10 times, 11-20 times, and >20 times per match) and 2) the number of previous concussions. Previous concussions included both soccer and nonsoccer related incidents, and, for the purposes of the questionnaire, a concussion was defined as having experienced loss of consciousness and/or amnesia after a head injury. The low-risk group was defined as those with no previous concussions and 0-5 headers per match; the high-risk group was defined as those with one or more previous concussions and $\geq 11$ headers per match. The remaining players were defined as a medium-risk group. Only samples from the low-risk and high-risk groups were analyzed.

\section{Blood samples and biochemical measurements}

All blood samples were collected from an antecubital vein, drawn into a standard gel $7 \mathrm{~mL}$ tube (BD Vacutainer blood collection tube, Becton Dickinson, Franklin Lakes, NJ, USA), 
1 before allowed to clot for $30 \mathrm{~min}$. They were then centrifuged (3000 $\mathrm{g}$ for $10 \mathrm{~min}$ ) before the

2 serum was divided between two $1.5 \mathrm{~mL}$ Eppendorf tubes (Eppendorf, Hamburg, Germany).

3 The samples were then frozen within $2 \mathrm{~h}$, after which one batch was used for the analyses in the original study (19) and the other batch was stored at $-80^{\circ} \mathrm{C}$ for later analyses.

During the summer of 2018 , the samples were thawed to $20^{\circ} \mathrm{C}$ and centrifuged (20 $000 \mathrm{~g}$ for $10 \mathrm{~min}$ ). Serum NfL and serum tau concentrations were then measured using ultrasensitive single molecule array technology (SIMOA-HD1, Quanterix Corporation, Lexington, MA, USA). Simoa ${ }^{\mathrm{TM}}$ NF-Light Advantage Kit and Simoa ${ }^{\mathrm{TM}}$ Tau Advantage Kit (Quanterix Corporation, Lexington, MA, USA) were used for NfL and tau protein quantification, respectively. Each measurement was done in duplicate, with a limit of detection of $38 \mathrm{fg} / \mathrm{mL}$ for serum $\mathrm{NfL}$ and $20 \mathrm{fg} / \mathrm{mL}$ for serum tau.

All analyses were performed blinded at the Institute of Neuroimmunology, Slovak Academy of Sciences, Bratislava, Slovakia, and in accordance with the manufacturer's recommendations.

\section{Statistical analyses}

Parametric tests were deemed appropriate for all groups at all time points. First, we screened for between-group and/or within-group differences using repeated measures ANOVA with Bonferroni correction for multiple group comparisons. If any significant differences were found, we further explored the dataset to locate their origin. For this, independent sample t-tests were used to evaluate between-group differences, and paired sample t-tests to evaluate within-group differences. When assessing the effects of short-term exposure (high-intensity exercise, repetitive headers and head-impact incidents), high-intensity exercise was considered reference. $\Delta \mathrm{B} 1$ and $\Delta \mathrm{B} 12$ values were calculated as the change in serum concentration from baseline to B1 and B12, respectively. For the effects of long-term exposure (number of previous concussions and headers), the low-risk group was considered as reference. All tests were two-sided using an $\alpha$-level of 0.05 for statistical significance. Multiple comparisons were not corrected for when using t-tests, as these analyses were considered exploratory. SPSS version 24 (IBM SPSS Statistics, IBM Corporation, Chicago, IL) was used for all statistical analyses. 


\section{Sample characteristics}

3 In total, we analyzed 354 samples for serum concentrations of NfL and tau proteins.

4 Mean \pm SD concentration of NfL was $6.8 \pm 2.6 \mathrm{pg} / \mathrm{mL}$; mean tau concentration was

$5 \quad 1.2 \pm 0.7 \mathrm{pg} / \mathrm{mL}$. The number of samples from the respective groups are shown in table 1.

6 Baseline characteristics of the groups are described in detail in the original study (19).

\section{Short-term effects on serum NfL}

8 Evaluating short-term effects on serum NfL levels, there were no between-group differences 9 (ANOVA, $\mathrm{p}=0.69$ ), nor any within-group differences (ANOVA, $\mathrm{p}=0.64$ ) (figure 1). For the

[Figure 1 near here]

\section{Short-term effects on serum tau}

Evaluating short-term effects on serum tau, there were both between-group differences (ANOVA, $\mathrm{p}<0.01$ ) and within-group differences (ANOVA, $\mathrm{p}<0.001$ ). When further exploring within-group differences, high-intensity exercise and repetitive headers displayed an increase in serum tau levels from baseline to $\mathrm{B} 1$ ( $\mathrm{p}<0.01$ for both); furthermore, both groups showed a subsequent decrease from B1 to B12 ( $<<0.001$ for high-intensity exercise and $\mathrm{p}<0.01$ for repetitive headers; figure 2). For the head-impact incidents, there were no within-group differences between any time points (figure 2). Compared to high-intensity exercise (reference), there were no between-group differences at any time point when evaluating the effects of repetitive headers; head-impact incidents displayed lower baseline and B1 values ( $\mathrm{p}<0.001$ for both), but there were no differences for B12, $\Delta \mathrm{B} 1$ and $\Delta \mathrm{B} 12$ values (figure 2). When comparing non-concussive and concussive head-impact incidents as sub-groups, there were no between-group differences for $\mathrm{B} 1, \mathrm{~B} 12, \Delta \mathrm{B} 1$ or $\Delta \mathrm{B} 12$ values. 
3 When comparing the low-risk and high-risk groups, we did not detect any long-term effects

\section{Long-term effects on serum NfL and serum tau} on serum NfL or tau concentrations (figure 3).

[Figure 3 near here]

\section{Discussion}

In this study, we investigated the potential short-term structural effects on the brain from head-impact exposure in elite soccer, using serum NfL and tau as biomarkers. Compared to high-intensity exercise as a reference, we found no evidence of acute axonal damage, neither from repetitive headers nor from head-impact incidents.

Furthermore, we assessed the potential long-term effects of previous, cumulative head-impact exposure. Comparing two groups with low vs. high exposure to previous concussions and headers, we found no evidence to indicate that previous head impacts initiate neurodegenerative processes or long-term changes, as detected by serum NfL and tau.

\section{Short-term effects on serum NfL}

In previous studies, NfL levels in blood have demonstrated diagnostic and prognostic utility in several scenarios related to head impacts in contact sports (20, 28-30). As an example, Shahim et al. (28) demonstrated that NfL can serve as a sensitive and dynamic biomarker for axonal injury after concussive injuries in boxing and ice hockey. Furthermore,

Rubin et al. (30) found that the frequency and magnitude of subconcussive head impacts in collegiate American football players were associated with changes in plasma NfL levels; this led the authors to speculate that NfL could be used to distinguish players sustaining more frequent and greater magnitude impacts from those with fewer and less severe impacts. Our results did not align with any of the findings described above. In fact, NfL levels seemed unaffected by all three conditions examined. 
We recognize several potential explanations for the apparent discrepancy. First, inherent differences in head-impact exposures between specific sports could mean that some impact types are more benign than others. Even though more than a third of the head-impact incidents in our study were classified as concussions, they were mostly in the milder end of the severity spectrum (19). Indeed, according to our injury-surveillance data, only two of the reported concussions were registered as time-loss injuries (table 1). Second, variations in study designs, such as the timing of blood sampling in relation to the exposures, will inevitably obscure what happens at other time points. Notably, Shahim et al. observed elevated NfL levels at $144 \mathrm{~h}$ after concussion (28), while we had a follow-up of no more than $12 \mathrm{~h}$. Therefore, our findings should be interpreted with caution. Nevertheless, even if future evidence should suggest that heading in soccer is harmful for the brain, our results document the limited utility of NfL as a biomarker for mild head impacts.

\section{Short-term effects on serum tau}

For serum tau, we observed dynamic short-term changes. However, these were only present for two of the conditions: repetitive headers and high-intensity exercise (figure 2). Importantly, tau levels remained unaffected by the head-impact incidents during regular league matches, also for impacts considered to be in the concussive range. As the session with high-intensity exercise yielded the most pronounced finding in our study, this suggests that the increase observed for the session with repetitive headers may simply be an exercise effect. Even though this session was designed to contain repetitive headers with otherwise low-intensity exercise, a certain amount of movement during the drills was inevitable.

If exercise causes an increase in tau levels, this should also have been the case for the head-impact incidents, as the players had already warmed up and played parts of a match (the incidents occurred approx. $30 \mathrm{~min}$ into the match on average). Indeed, we did observe a trend towards an increase $1 \mathrm{~h}$ after exposure also in this group (figure 2). One potential cause for a non-significant difference is that blood samples from this group were drawn later than for the reference, $77 \mathrm{~min}$ vs. $26 \mathrm{~min}$ (19). This time window could have allowed levels to fall.

Interestingly, we also observed some unexpected between-group differences, with head-impact incidents yielding lower absolute values compared to the reference at both baseline and B1. We can only speculate as to why this was the case. One possible explanation is that baseline tau levels fluctuate throughout the season. Specifically, the timing of the exercise sessions differed between the three teams, which might represent a critical source of 
heterogeneity; one team completed them during the middle of the pre-season, one team at the end of the pre-season, and one team right after the end of the season (all players had baseline sampling performed in the morning before the first of the two sessions). In contrast, the baseline samples for the head-impact incidents were all collected in the pre-season.

In summary, we found no evidence suggestive of structural neuronal damage, but revealed a substantial confounding effect from exercise. Indeed, this is in line with recent work by Kawata et al. (31). Examining collegiate American football players before and after a series of practices, they found that plasma tau levels increased after all the sessions; however, these changes were independent of subconcussive head impacts as measured by mouth-guard accelerometers.

Our study highlights several important limitations of measuring total tau as a biomarker for head injuries in the lower severity spectrum. Importantly, an appropriate control group should be included in similar studies in contact sports, as physical activity needs to be controlled for. This is also supported by findings of Gill et al. (32), describing elevated tau levels in response to exercise. What actually accounts for this remains unknown, but factors such as increased neuronal activity (33), clearance via the glymphatic system (34), and changes in the permeability of the blood-brain barrier $(35,36)$ are thought to play a role. Furthermore, other sources of tau protein release have been described, such as from the enteric nervous system (37). The ultrasensitive assay we used in this study does not distinguish between specific tau isoforms and their origins.

\section{Long-term effects of previous head-impact exposure}

NfL levels in blood have been linked to neurodegeneration, irrespective of the underlying cause (21), and elevated levels in e.g. Alzheimer's disease can appear years to decades before onset of neurological symptoms (22). Studies on military personnel have also demonstrated long-term elevations of circulating tau associated with multiple TBIs (38).

Di Battista et al. (39) have demonstrated similar findings associated with participation in contact sports. It is important to point out, however, that other studies have failed to demonstrate any effects on NfL and tau levels from previous head-impact exposure and contact sport participation $(30,31)$.

Therefore, as a secondary aim in our study, we wanted to explore the long-term effects of previous concussions and headers, by comparing two groups characterized by differences in cumulative exposure (low-risk vs. low-risk). We wanted to assess whether having a 
positive concussion history, in combination with a playing style with frequent heading

2 behavior in matches, would lead to sustained elevations in serum levels of NfL and/or tau proteins. We did not observe such changes. Despite a trend towards higher NfL levels in the high-risk group (figure 3), the effect size was negligible and we did not demonstrate any between-group differences.

We recognize at least three possible explanations for these findings. First, the previous head-impact exposure in the high-risk group was not deleterious to the brain, implying that reparative processes have been able to compensate and thereby 'keep up with' the forces exerted upon the nervous tissue over time. Second, and in contrast to the first, the biomarkers were not sufficiently sensitive to detect any deleterious effects of the previous exposure. Third, even though self-reported measures of head-impact exposure in contact sports have been shown to be useful (40), there is inevitably a high risk of bias (41). Also, in the questionnaire concussion was defined using the diagnostic criteria commonly used by medical personnel in Norway at the time (i.e. loss of consciousness/amnesia was required). Thereby using a stricter definition than the one currently used in sports (11), the low-risk group might also have sustained concussions that had not been recognized and therefore not reported. Consequently, and despite our best efforts to trichotomize the groups appropriately, there could be a considerable overlap between the high- and low-risk groups, which might conceal differences. Considering such limitations, these data should be interpreted with caution.

\section{Methodological considerations}

Relevant to all the investigations we conducted in this study, it should be noted that any one clinical biomarker reflects only a small piece of a complex pathophysiological puzzle. Notably, in the absence of other investigations such as neuroimaging, the limited sensitivity and specificity of serum NfL and tau to pick up neuronal damage might yield false negative findings. Therefore, it remains unknown to what extent the results are best explained by the exposure being benign or the biomarkers being inadequate. We used a highly sensitive and widely recognized method for both serum NfL and serum tau measurements. However, Rubenstein et al. (42) have already demonstrated how hyperphosporylated tau levels in blood outperforms total tau in both diagnosis and prognosis of TBI. In general, future studies should strive to utilize and advance the most sensitive and specific assays available.

Furthermore, the quantification of head impacts warrants careful consideration when exploring the effects of head-impact exposure in contact sports. In our study, the short-term 
exposures (i.e. repetitive headers and head-impact incidents) were only confirmed visually; therefore, we could not report on the objective impact forces involved (using e.g. accelerometers). This limitation also applies to the long-term exposure, which was quantified using self-report.

Regarding the absolute serum NfL and tau values, we hesitate in comparing our results directly to those of others. The main reason is that we do not know the impact of methodological differences between studies, such as the on-field blood sample collection procedures and subsequent management (e.g. duration of centrifugation). Concerning long-term storage of frozen blood samples, there are three main reasons why we deem our results to be valid: (1) previous research suggests that $\mathrm{NfL}$ and tau integrity is stable in relation to both long-term storage and multiple freeze-thaw cycles (43); (2) the serum concentrations were within the expected range when comparing with the data supplied by the producer of the kits; and (3) any degradation would affect the samples evenly between and within groups, thereby minimizing its impact on our results.

Lastly, as we did not correct for multiple testing when exploring specific within- and between-group differences, the results and conclusions from this part should be considered exploratory. We encourage others to further examine the time course of changes in the biomarkers and their relationship with head-impact characteristics.

\section{Conclusion}

This study demonstrates that serum NfL and tau were unaffected by head impacts in soccer. Furthermore, it shows how circulating tau levels rise immediately after exercise, with a rapid return to resting values, whereas NfL levels remain unchanged. Our findings highlight important characteristics and limitations when using NfL or tau as biomarkers in sports. 


\section{Acknowledgements}

2 The authors are grateful to all the players, medical staff and research personnel who took part

3 in the original study. We thank Jiri Dvorak and Marianne Jochum for their contributions in

4 the original study design and analyses. For their outstanding help in data collection and blood

5 sampling, we also thank Jostein Steene-Johannesen, Grete Steene-Johannesen, Hilde

6 Mikkelsen Bakka, John Bjørneboe and Torbjørn Soligard.

\section{Author disclosure statement}

8 This work was part of the study RepImpact, and was funded by ERA-NET NEURON and The

9 Research Council of Norway. The original study was funded by the International Federation

10 of Association Football Medical Assessment and Research Center. The Oslo Sports Trauma

11 Research Center has been established at the Norwegian School of Sport Sciences through generous grants from the Royal Norwegian Ministry of Culture, the South-Eastern Norway Regional Health Authority the International Olympic Committee, the Norwegian Olympic Committee and Confederation of Sport, and Norsk Tipping AS. No other competing financial interests exist. 


\section{References}

1. Asken BM, Sullan MJ, DeKosky ST, Jaffee MS, Bauer RM. Research gaps and controversies in chronic traumatic encephalopathy: A review. JAMA neurology. 2017. doi: 10.1001/jamaneurol.2017.2396.

2. McKee AC, Cantu RC, Nowinski CJ, Hedley-Whyte ET, Gavett BE, Budson AE, et al. Chronic traumatic encephalopathy in athletes: progressive tauopathy after repetitive head injury. J Neuropathol Exp Neurol. 2009;68(7):709-35. Epub 2009/06/19. doi: 10.1097/NEN.0b013e3181a9d503. PubMed PMID: 19535999; PubMed Central PMCID: PMCPMC2945234.

3. Mez J, Daneshvar DH, Kiernan PT, Abdolmohammadi B, Alvarez VE, Huber BR, et al. Clinicopathological Evaluation of Chronic Traumatic Encephalopathy in Players of American Football. JAMA. 2017;318(4):360-70. Epub 2017/07/26. doi: 10.1001/jama.2017.8334. PubMed PMID: 28742910.

4. Chrisman SP, Mac Donald CL, Friedman S, Andre J, Rowhani-Rahbar A, Drescher S, et al. Head Impact Exposure During a Weekend Youth Soccer Tournament. J Child Neurol. 2016;31(8):971-8. Epub 2016/03/10. doi: 10.1177/0883073816634857. PubMed PMID: 26951540. 5. Press JN, Rowson S. Quantifying Head Impact Exposure in Collegiate Women's Soccer. Clin J Sport Med. 2017;27(2):104-10. Epub 2016/03/16. doi: 10.1097/jsm.0000000000000313. PubMed PMID: 26978008.

6. Sandmo SB, McIntosh AS, Andersen TE, Koerte IK, Bahr R. Evaluation of an In-Ear Sensor for Quantifying Head Impacts in Youth Soccer. Am J Sports Med. 2019:363546519826953. Epub 2019/02/26. doi: 10.1177/0363546519826953. PubMed PMID: 30802147.

7. Andersen TE, Arnason A, Engebretsen L, Bahr R. Mechanisms of head injuries in elite football. Br J Sports Med. 2004;38(6):690-6. Epub 2004/11/25. doi: 10.1136/bjsm.2003.009357. PubMed PMID: 15562161; PubMed Central PMCID: PMCPMC1724962.

8. Kontos AP, Braithwaite R, Chrisman SP, McAllister-Deitrick J, Symington L, Reeves VL, et al. Meta-analytical review of the effects of football heading. Br J Sports Med. 2016. Epub 2016/12/23. doi: 10.1136/bjsports-2016-096276. PubMed PMID: 28003239.

9. Rodrigues AC, Lasmar RP, Caramelli P. Effects of Soccer Heading on Brain Structure and Function. Front Neurol. 2016;7:38. Epub 2016/04/06. doi: 10.3389/fneur.2016.00038. PubMed PMID: 27047444; PubMed Central PMCID: PMCPMC4800441.

10. Tarnutzer AA, Straumann D, Brugger P, Feddermann-Demont N. Persistent effects of playing football and associated (subconcussive) head trauma on brain structure and function: a systematic review of the literature. Br J Sports Med. 2016. Epub 2016/11/07. doi: 10.1136/bjsports-2016-096593. PubMed PMID: 27815240.

11. McCrory P, Meeuwisse W, Dvorak J, Aubry M, Bailes J, Broglio S, et al. Consensus statement on concussion in sport-the 5th international conference on concussion in sport held in Berlin, October 2016. Br J Sports Med. 2017. Epub 2017/04/28. doi: 10.1136/bjsports-2017-097699. PubMed PMID: 28446457.

12. Bigler ED, Maxwell WL. Neuropathology of mild traumatic brain injury: relationship to neuroimaging findings. Brain imaging and behavior. 2012;6(2):108-36. Epub 2012/03/22. doi: 10.1007/s11682-011-9145-0. PubMed PMID: 22434552.

13. Giza CC, Hovda DA. The Neurometabolic Cascade of Concussion. Journal of athletic training. 2001;36(3):228-35. Epub 2003/08/26. PubMed PMID: 12937489; PubMed Central PMCID: PMCPMC155411.

14. Zetterberg H, Blennow K. Fluid biomarkers for mild traumatic brain injury and related conditions. Nat Rev Neurol. 2016;12(10):563-74. Epub 2016/09/17. doi: 10.1038/nrneurol.2016.127. PubMed PMID: 27632903.

15. Wang KK, Yang Z, Zhu T, Shi Y, Rubenstein R, Tyndall JA, et al. An update on diagnostic and prognostic biomarkers for traumatic brain injury. Expert Rev Mol Diagn. 2018;18(2):165-80. Epub 2018/01/18. doi: 10.1080/14737159.2018.1428089. PubMed PMID: 29338452; PubMed Central PMCID: PMCPMC6359936.

16. Unden L, Calcagnile O, Unden J, Reinstrup P, Bazarian J. Validation of the Scandinavian guidelines for initial management of minimal, mild and moderate traumatic brain injury in adults. 
BMC Med. 2015;13:292. Epub 2015/12/10. doi: 10.1186/s12916-015-0533-y. PubMed PMID: 26645914; PubMed Central PMCID: PMCPMC4673733.

17. Bazarian JJ, Biberthaler P, Welch RD, Lewis LM, Barzo P, Bogner-Flatz V, et al. Serum GFAP and UCH-L1 for prediction of absence of intracranial injuries on head CT (ALERT-TBI): a multicentre observational study. Lancet Neurol. 2018;17(9):782-9. Epub 2018/07/29. doi: 10.1016/s1474-4422(18)30231-x. PubMed PMID: 30054151.

18. McCrea M, Meier T, Huber D, Ptito A, Bigler E, Debert CT, et al. Role of advanced neuroimaging, fluid biomarkers and genetic testing in the assessment of sport-related concussion: a systematic review. Br J Sports Med. 2017. Epub 2017/04/30. doi: 10.1136/bjsports-2016-097447. PubMed PMID: 28455364.

19. Straume-Naesheim TM, Andersen TE, Jochum M, Dvorak J, Bahr R. Minor head trauma in soccer and serum levels of S100B. Neurosurgery. 2008;62(6):1297-305; discussion 305-6. Epub 2008/10/01. doi: 10.1227/01.neu.0000333301.34189.3d. PubMed PMID: 18824996.

20. Shahim P, Tegner Y, Marklund N, Blennow K, Zetterberg H. Neurofilament light and tau as blood biomarkers for sports-related concussion. Neurology. 2018. Epub 2018/04/15. doi: 10.1212/wnl.0000000000005518. PubMed PMID: 29653990.

21. Khalil M, Teunissen CE, Otto M, Piehl F, Sormani MP, Gattringer T, et al. Neurofilaments as biomarkers in neurological disorders. Nat Rev Neurol. 2018;14(10):577-89. Epub 2018/09/02. doi: 10.1038/s41582-018-0058-z. PubMed PMID: 30171200.

22. Preische O, Schultz SA, Apel A, Kuhle J, Kaeser SA, Barro C, et al. Serum neurofilament dynamics predicts neurodegeneration and clinical progression in presymptomatic Alzheimer's disease. Nat Med. 2019;25(2):277-83. Epub 2019/01/22. doi: 10.1038/s41591-018-0304-3. PubMed PMID: 30664784; PubMed Central PMCID: PMCPMC6367005.

23. Straume-Naesheim TM, Andersen TE, IM KH, McIntosh AS, Dvorak J, Bahr R. Do minor head impacts in soccer cause concussive injury? A prospective case-control study. Neurosurgery. 2009;64(4):719-25; discussion 25. Epub 2009/04/08. doi: 10.1227/01.neu.0000340681.12949.6d. PubMed PMID: 19349829.

24. Straume-Naesheim TM, Andersen TE, Dvorak J, Bahr R. Effects of heading exposure and previous concussions on neuropsychological performance among Norwegian elite footballers. $\mathrm{Br} \mathbf{J}$ Sports Med. 2005;39 Suppl 1:i70-7. Epub 2005/07/28. doi: 10.1136/bjsm.2005.019646. PubMed PMID: 16046359; PubMed Central PMCID: PMCPMC1765315.

25. Andersen TE, Larsen O, Tenga A, Engebretsen L, Bahr R. Football incident analysis: a new video based method to describe injury mechanisms in professional football. Br J Sports Med. 2003;37(3):226-32. Epub 2003/06/05. PubMed PMID: 12782547; PubMed Central PMCID: PMCPMC1724642.

26. Aubry M, Cantu R, Dvorak J, Graf-Baumann T, Johnston K, Kelly J, et al. Summary and agreement statement of the First International Conference on Concussion in Sport, Vienna 2001. Recommendations for the improvement of safety and health of athletes who may suffer concussive injuries. Br J Sports Med. 2002;36(1):6-10. Epub 2002/02/28. PubMed PMID: 11867482; PubMed Central PMCID: PMCPMC1724447.

27. Andersen TE, Tenga A, Engebretsen L, Bahr R. Video analysis of injuries and incidents in Norwegian professional football. Br J Sports Med. 2004;38(5):626-31. Epub 2004/09/25. doi: 10.1136/bjsm.2003.007955. PubMed PMID: 15388553; PubMed Central PMCID: PMCPMC1724925. 28. Shahim P, Zetterberg H, Tegner Y, Blennow K. Serum neurofilament light as a biomarker for mild traumatic brain injury in contact sports. Neurology. 2017;88(19):1788-94. Epub 2017/04/14. doi: 10.1212/wnl.0000000000003912. PubMed PMID: 28404801; PubMed Central PMCID: PMCPMC5419986.

29. Shahim P, Gren M, Liman V, Andreasson U, Norgren N, Tegner Y, et al. Serum neurofilament light protein predicts clinical outcome in traumatic brain injury. Sci Rep. 2016;6:36791. Epub 2016/11/08. doi: 10.1038/srep36791. PubMed PMID: 27819296; PubMed Central PMCID: PMCPMC5098187 N.N. is employed by UmanDiagnostics. H.Z. and K.B. are co-founders of Brain Biomarker Solutions in Gothenburg AB, a GU Venture-based platform company at the University of Gothenburg, Sweden.

30. Rubin LH, Tierney R, Kawata K, Wesley L, Lee JH, Blennow K, et al. NFL blood levels are moderated by subconcussive impacts in a cohort of college football players. Brain Inj. 
2019;33(4):456-62. Epub 2019/02/20. doi: 10.1080/02699052.2019.1565895. PubMed PMID: 30776989 .

31. Kawata K, Rubin LH, Wesley L, Lee JH, Sim T, Takahagi M, et al. Acute Changes in Plasma Total Tau Levels Are Independent of Subconcussive Head Impacts in College Football Players. J Neurotrauma. 2018;35(2):260-6. Epub 2017/10/28. doi: 10.1089/neu.2017.5376. PubMed PMID: 29073820.

32. Gill J, Merchant-Borna K, Jeromin A, Livingston W, Bazarian J. Acute plasma tau relates to prolonged return to play after concussion. Neurology. 2017;88(6):595-602. Epub 2017/01/08. doi: 10.1212/wnl.0000000000003587. PubMed PMID: 28062722; PubMed Central PMCID: PMCPMC5304458.

33. Knaepen K, Goekint M, Heyman EM, Meeusen R. Neuroplasticity - exercise-induced response of peripheral brain-derived neurotrophic factor: a systematic review of experimental studies in human subjects. Sports Med. 2010;40(9):765-801. Epub 2010/08/24. doi: 10.2165/11534530000000000-00000. PubMed PMID: 20726622.

34. Iliff JJ, Chen MJ, Plog BA, Zeppenfeld DM, Soltero M, Yang L, et al. Impairment of glymphatic pathway function promotes tau pathology after traumatic brain injury. J Neurosci. 2014;34(49):16180-93. Epub 2014/12/05. doi: 10.1523/jneurosci.3020-14.2014. PubMed PMID: 25471560; PubMed Central PMCID: PMCPMC4252540.

35. Sharma HS, Cervos-Navarro J, Dey PK. Increased blood-brain barrier permeability following acute short-term swimming exercise in conscious normotensive young rats. Neurosci Res. 1991;10(3):211-21. Epub 1991/04/01. PubMed PMID: 1650437.

36. Watson P, Shirreffs SM, Maughan RJ. Blood-brain barrier integrity may be threatened by exercise in a warm environment. Am J Physiol Regul Integr Comp Physiol. 2005;288(6):R1689-94. Epub 2005/01/15. doi: 10.1152/ajpregu.00676.2004. PubMed PMID: 15650123.

37. Lionnet A, Wade MA, Corbille AG, Prigent A, Paillusson S, Tasselli M, et al. Characterisation of tau in the human and rodent enteric nervous system under physiological conditions and in tauopathy. Acta neuropathologica communications. 2018;6(1):65. Epub 2018/07/25. doi: 10.1186/s40478-018-0568-3. PubMed PMID: 30037345; PubMed Central PMCID: PMCPMC6055332.

38. Olivera A, Lejbman N, Jeromin A, French LM, Kim HS, Cashion A, et al. Peripheral Total Tau in Military Personnel Who Sustain Traumatic Brain Injuries During Deployment. JAMA neurology. 2015;72(10):1109-16. Epub 2015/08/04. doi: 10.1001/jamaneurol.2015.1383. PubMed PMID: 26237304.

39. Di Battista AP, Rhind SG, Richards D, Churchill N, Baker AJ, Hutchison MG. Altered Blood Biomarker Profiles in Athletes with a History of Repetitive Head Impacts. PLoS One. 2016;11(7):e0159929. Epub 2016/07/28. doi: 10.1371/journal.pone.0159929. PubMed PMID: 27458972; PubMed Central PMCID: PMCPMC4961456.

40. Montenigro PH, Alosco ML, Martin BM, Daneshvar DH, Mez J, Chaisson CE, et al. Cumulative Head Impact Exposure Predicts Later-Life Depression, Apathy, Executive Dysfunction, and Cognitive Impairment in Former High School and College Football Players. J Neurotrauma. 2017;34(2):328-40. Epub 2016/04/01. doi: 10.1089/neu.2016.4413. PubMed PMID: 27029716; PubMed Central PMCID: PMCPMC5220530.

41. Catenaccio E, Caccese J, Wakschlag N, Fleysher R, Kim N, Kim M, et al. Validation and calibration of HeadCount, a self-report measure for quantifying heading exposure in soccer players. Res Sports Med. 2016;24(4):416-25. Epub 2016/10/30. doi: 10.1080/15438627.2016.1234472. PubMed PMID: 27788599; PubMed Central PMCID: PMCPMC5144162.

42. Rubenstein R, Chang B, Yue JK, Chiu A, Winkler EA, Puccio AM, et al. Comparing Plasma Phospho Tau, Total Tau, and Phospho Tau-Total Tau Ratio as Acute and Chronic Traumatic Brain Injury Biomarkers. JAMA neurology. 2017. Epub 2017/07/25. doi: 10.1001/jamaneurol.2017.0655. PubMed PMID: 28738126.

43. Bergman L, Zetterberg H, Kaihola H, Hagberg H, Blennow K, Akerud H. Blood-based cerebral biomarkers in preeclampsia: Plasma concentrations of NfL, tau, S100B and NSE during pregnancy in women who later develop preeclampsia - A nested case control study. PLoS One. 2018;13(5):e0196025. Epub 2018/05/03. doi: 10.1371/journal.pone.0196025. PubMed PMID: 29719006; PubMed Central PMCID: PMCPMC5931625. 
1 Figure 1. Serum NfL values at baseline, 1 hour (B1) and 12 hours (B12) after exposure to

2 high-intensity exercise, repetitive headers and a head-impact incident in a match. Values are 3 presented as means and error bars represent $95 \%$ confidence intervals.

4 Figure 2. Serum tau values at baseline, 1 hour (B1) and 12 hours (B12) after exposure to 5 high-intensity exercise, repetitive headers and a head-impact incident in a match. Values are 6 presented as means and error bars represent $95 \%$ confidence intervals.

7 Figure 3. The long-term effects of previous head-impact exposure on serum tau and serum 8 NfL in elite soccer players at pre-season. The two groups are categorized based on their 9 previously low and high levels of exposure to concussions and soccer headers. Values are 10 presented as means and error bars represent $95 \%$ confidence intervals. 
Table 1. Overview of the number of samples analyzed from the different groups at each time point.

\begin{tabular}{|c|c|c|c|c|c|}
\hline & \multicolumn{3}{|c|}{$\underline{\text { Short-term exposure }}$} & \multicolumn{2}{|c|}{$\underline{\text { Long-term exposure }}$} \\
\hline & $\begin{array}{l}\text { High-intensity } \\
\text { exercise }\end{array}$ & $\begin{array}{l}\text { Repetitive } \\
\text { headers }\end{array}$ & $\begin{array}{c}\text { Head } \\
\text { impacts }\end{array}$ & $\begin{array}{l}\text { High-risk } \\
\text { group* }\end{array}$ & $\begin{array}{c}\text { Low-risk } \\
\text { group* }\end{array}$ \\
\hline Baseline samples, BL (n) & 47 & 47 & 35 & 23 & 57 \\
\hline 1h post-expoure, B1 (n) & 37 & 38 & 35 & $N / A$ & $N / A$ \\
\hline Concussions $* *(n)$ & $N / A$ & $N / A$ & 14 & & \\
\hline Non-concussions ( $n$ ) & $N / A$ & $N / A$ & 21 & & \\
\hline $12 \mathrm{~h}$ post-exposure, $\mathrm{B} 12(\mathrm{n})$ & 33 & 30 & 19 & N/A & $N / A$ \\
\hline Concussions ${ }^{* *}(n)$ & $N / A$ & $N / A$ & 8 & & \\
\hline Non-concussions (n) & $N / A$ & N/A & 11 & & \\
\hline
\end{tabular}

*Risk groups related to long-term exposure were based on self-reported previous head-impact exposure, including average headers per match and concussion history.

**For the head-impact group, concussions were classified according to the presence of symptoms, based on the Vienna concussion definition (2001). Two of the 14 cases were reported as time-loss injuries. 